\title{
New Aspects for the Treatment of Cardiac Diseases Based on the Diversity of Functional Controls on Cardiac Muscles: Novel Approaches for the Diagnosis and Treatment of Cardiac Diseases
}

\author{
Takeshi Suzuki ${ }^{1, *}$ and Hikaru Tanaka ${ }^{2}$ \\ ${ }^{1}$ Faculty of Pharmacy, Division of Basic Biological Sciences, Keio University, Tokyo 105-8512, Japan \\ ${ }^{2}$ Department of Pharmacology, Toho University Faculty of Pharmaceutical Sciences, Chiba 274-8510, Japan
}

Received October 16, 2008; Accepted October 24, 2008

Keywords: arrhythmia, cardiac muscle, $\mathrm{Ca}^{2+}$, cardiac disease, heart failure

The rise in the number of patients with cardiac diseases in the coming aging society would increase the needs for accurate diagnosis and therapeutics for cardiac diseases. The function of the heart is supported by excitation and contraction of the myocardium, which are complex processes involving generation of the action potential, $\mathrm{Ca}^{2+}$ mobilization, and the activation of the contractile proteins. These are supported by myocardial energy metabolism and are under neurohumoral control. Under pathological conditions, alterations occur in the proteins involved in myocardial contraction and also in the neurohumoral status. Many biomarkers have been demonstrated to be useful for the diagnosis of cardiac diseases. Under the atrial fibrillation condition, changes in interleukin-6 $(1,2)$, C-reactive protein (CRP) (2), atrial natriuretic peptide (ANP), and brain natriuretic peptide (BNP) (3) were reported. Under the ventricular tachycardia and premature contraction, changes in BNP and CRP are also reported $(4,5)$. Under the heart failure condition, changes in both facilitating markers (e.g., rennin - angiotensin - aldosterone system, sympathetic nervous system) and protective markers (e.g., ANP, BNP) are reported $(6,7)$. These may be either the result of the pathological changes forming a vicious cycle or rather a reflection of some compensatory mechanism against the pathological changes.

Concerning pharmacological treatment of cardiac disorders, new types of therapeutic agents such as antibodies and nucleic acids are now under development in addition to the standard drugs consisting of low molecular weight compounds. Furthermore, in the near future, molecular biology and regenerative techniques would enable manipulation of the functional proteins

*Corresponding author. Suzuki-tk@pha.keio.ac.jp Published online in J-STAGE on March 7, 2009 (in advance) doi: 10.1254 /jphs.08R21FM and replacement of the damaged myocardium with regenerated myocardium. Under such circumstances, an understanding of the optimum balance of function in the heart, as well as information on the individual elements, is important for the development of an effective therapeutic strategy. The following reviews summarized below provide a basis for such comprehensive understanding of the heart.

In "Diversity in the excitation-contraction mechanisms of the heart", Hikaru Tanaka (Toho University Faculty of Pharmaceutical Sciences) et al. (8) presented an overview of myocardial diversity among species, developmental stage, and the region of the heart. Diversity in the ion channels results in diversity in action potential waveform and excitability of the myocardium. The $\mathrm{Ca}^{2+}$ release from the sarcoplasmic reticulum, which is the major determinant of myocardial contractile function, is largely affected by the action potential waveformthrough the activity of the $\mathrm{Na}^{+} / \mathrm{Ca}^{2+}$ exchanger. Myocardial contraction is dependent on the energy metabolism and is under neurohumoral regulation. A precise and comprehensive understanding of each type of myocardium including various pathological models is essential for the clarification of the general principle of myocardial regulation and the development of novel therapeutic agents.

In "Acute effects of female hormones on cardiac ion channels and cardiac repolarization", Junko Kurokawa (Medical Research Institute, Tokyo Medical and Dental University) et al. (9) introduced their recent results showing that physiological levels of ovarian steroids, estradiol and progesterone, have reverse effects on myocardial repolarization, which may create cyclic variation of cardiac repolarization in women. Estradiol prolongs while progesterone shortens action potential duration and QT interval through direct effects on cardiac ion channels, a regulatory mechanism distinct 
from the well-characterized transcriptional regulation by steroid hormones. These findings may explain dynamic changes of arrhythmia risk in women during the menstrual cycle and around the delivery, and they can be a clue to avoid the potentially lethal arrhythmia in long-QT syndromes.

In "Mitochondrial ion channels and cardioprotection", Hirofumi Nishida (Chiba University Graduate School of Medicine) et al. (10) summarized their recent findings on mitochondrial ATP-sensitive $\mathrm{K}^{+}$channels and $\mathrm{Ca}^{2+}$-activated $\mathrm{K}^{+}$channels. Mitochondrial $\mathrm{K}^{+}$influx through these channels occurs independently of each other and confers cardioprotection in a similar manner. Adenosine and bradykinin stimulate the opening of the mitochondrial ATP-sensitive $\mathrm{K}^{+}$channels through activation of protein kinase $\mathrm{C}$ while adrenomedulin stimulates the opening of mitochondrial $\mathrm{Ca}^{2+}$-activated $\mathrm{K}^{+}$channels through activation of protein kinase $\mathrm{C}$. These endogenous substances have cardioprotective effects such as reduction of infarction size after ischemiareperfusion injury. Thus, protection of the mitochondria through mitochondrial potassium channels appears to be a promising strategy for cardioprotection.

In "The regulatory mechanisms of cardiac innervation and their critical roles in cardiac performance", Masaki Ieda and Keiichi Fukuda (Keio University School of Medicine) (11) presented their recent findings on the molecular mechanisms underlying the normal development of cardiac innervation and demonstrated the importance of innervation in the maintenance of normal cardiac function. Endothelin-1 plays a critical role in the development of cardiac innervation through upregulation of nerve growth factor, a neural chemoattractant essential for the survival and differentiation of peripheral sensory and sympathetic neurons. The expression pattern of Sema3a, a neural chemorepellent, was found to be a major determinant of the epicardial-toendocardial gradient of sympathetic innervation. Pertubation of Sema3a expression leads to disruption of the sympathetic innervation pattern and abnormal excitability of the ventricle.

In "Effects of targeted disruption of the type 5 adenylyl cyclase gene", Satoshi Okumura (Yokohama City University Graduate School of Medicine) et al. (12) presented their recent findings obtained with type 5 adenylyl cyclase knockout mouse. The results obtained suggested that among the adenylyl cyclase isoforms present in the heart, type 5 is potentially important in the autonomic regulation and $\mathrm{Ca}^{2+}$-mediated regulation of the heart. Type 5 adenylyl cyclase also appears to be involved in the induction of myocardial damage in response to pressure overload and catecholamine stress. Thus, concerning the pharmacological treatment of heart failure, selective suppression of type 5 adenylyl cyclase activity may have an advantage over the current $\beta$ - adrenoceptor blockade, which suppresses the entire $\beta$ adrenergic signaling pathway.

Observations shown in the above articles would indicate to us several novel approaches for the accurate diagnosis and therapeutics for cardiac diseases.

(This and the following articles were based on the symposium on this topic at the 81st Annual Meeting of The Japanese Pharmacological Society held on March 17 to 19, 2008 in Yokohama, Japan)

\section{References}

1 Conway DSG, Buggins P, Hughes E, Lip GYH. Prognostic significance of raised plasma levels of interleukin-6 and Creactive protein in atrial fibrillation. Am Heart J. 2004;148:462466.

2 Gedikli O, Dogan A, Altuntas A, Ozaydin M, Akturk O, Acar G. Inflammatory markers according to types of atrial fibrillation. Int J Cardiol. 2007;120:193-197.

3 Dixen U, Ravn L, Soeby-Rasmussen C, Paulsen AW, Parner J, Frandsen E, et al. Raised plasma aldosterone and natriuretic peptides in atrial fibrillation. Cardiology. 2007;108:35-39.

4 Blangy H, Sadoul N, Dousset B, Radauceanu A, Fay R, Aliot E, et al. Serum BNP, hs-C-reactive protein, procollagen to assess the risk of ventricular tachycardia in ICD recipients after myocardial infarction. Europace. 2007;8:724-729.

5 Berger R, Huelsman M, Strecker K, Bojic A, Moser P, Stanek B, et al. B-type natriuretic peptide predicts sudden death in patients with chronic heart failure. Circulation. 2002;105:2392-2397.

6 Maeda M, Tsutamoto T, Wada A, Hisanaga T, Kinoshita M. Plasma brain natriuretic peptide as a biochemical marker of high left ventricular end-diastlic pressure in patients with symptomatic left ventricular dysfunction. Am Heart J. 1998;135: 825-832.

7 Anand IS, Latini R, Florea VG, Kuskowski MA, Rector T, Masson S, et al. C-reactive protein in heart failure: prognostic value and the effect of valsartan. Circulation. 2005;112:14281434.

8 Tanaka H, Namekata I, Nouchi H, Shigenobu K, Kawanishi T, Takahara A. New aspects for the treatment of cardiac diseases based on the diversity of functional controls on cardiac muscles: Diversity in the excitation-contraction mechanisms of the heart. J Pharmacol Sci. 2009; 109:327-333.

9 Kurokawa J, Suzuki T, Furukawa T. New aspects for the treatment of cardiac diseases based on the diversity of functional controls on cardiac muscles: Acute effects of female hormones on cardiac ion channels and cardiac repolarization. J Pharmacol Sci. 2009;109:334-340.

10 Nishida H, Sato T, Ogura T, Nakaya H. New aspects for the treatment of cardiac diseases based on the diversity of functional controls on cardiac muscles: Mitochondrial ion channels and cardioprotection. J Pharmacol Sci. 2009;109:341-347.

11 Ieda M, Fukuda K. New aspects for the treatment of cardiac diseases based on the diversity of functional controls on cardiac muscles: The regulatory mechanisms of cardiac innervation and their critical roles in cardiac performance. J Pharmacol Sci. 2009;109:348-353.

12 Okumura S, Suzuki S, Ishikawa Y. New aspects for the treatment of cardiac diseases based on the diversity of functional controls on cardiac muscles: Effects of targeted disruption of the type 5 adenylyl cyclase gene. J Pharmacol Sci. 2009;109:354-359. 